\title{
Multivalent ligands control stem cell behaviour in vitro and in vivo
}

\author{
Anthony Conway1, Tandis Vazin"1,2, Dawn P. Spelke², Nikhil A. Rode ${ }^{3}$, Kevin E. Healy ${ }^{2,3}$, Ravi S. Kane \\ and David V. Schaffer ${ }^{1,2 \star}$
}

There is broad interest in designing nanostructured materials that can interact with cells and regulate key downstream functions $^{1-7}$. In particular, materials with nanoscale features may enable control over multivalent interactions, which involve the simultaneous binding of multiple ligands on one entity to multiple receptors on another and are ubiquitous throughout biology ${ }^{8-10}$. Cellular signal transduction of growth factor and morphogen cues (which have critical roles in regulating cell function and fate) often begins with such multivalent binding of ligands, either secreted or cell-surface-tethered to target cell receptors, leading to receptor clustering ${ }^{11-18}$. Cellular mechanisms that orchestrate ligand-receptor oligomerization are complex, however, so the capacity to control multivalent interactions and thereby modulate key signalling events within living systems is currently very limited. Here, we demonstrate the design of potent multivalent conjugates that can organize stem cell receptors into nanoscale clusters and control stem cell behaviour in vitro and in vivo. The ectodomain of ephrin-B2, normally an integral membrane protein ligand, was conjugated to a soluble biopolymer to yield multivalent nanoscale conjugates that potently induce signalling in neural stem cells and promote their neuronal differentiation both in culture and within the brain. Super-resolution microscopy analysis yielded insights into the organization of the receptor-ligand clusters at the nanoscale. We also found that synthetic multivalent conjugates of ephrin-B1 strongly enhance human embryonic and induced pluripotent stem cell differentiation into functional dopaminergic neurons. Multivalent bioconjugates are therefore powerful tools and potential nanoscale therapeutics for controlling the behaviour of target stem cells in vitro and in vivo.

Adult neural stem cells (NSCs) are an important class of therapeutically relevant cells that persist in specific regions of the mammalian brain, and have the capacity to generate new neurons and glia throughout life ${ }^{19}$. Human pluripotent stem cells (hPSCs), which include human embryonic stem cells (hESCs) and induced pluripotent stem cells (hiPSCs), have the capacity to differentiate into all cells of the adult body and therefore offer broad potential for cell replacement therapy and modelling of human disease. We recently found that ephrin-Eph signalling regulates both the neuronal differentiation of adult hippocampal NSCs ${ }^{19}$ and the differentiation of hESCs into dopaminergic neurons ${ }^{20}$, cells lost in Parkinson's disease. The design of molecules that regulate ephrinEph signalling in NSCs and hPSCs could therefore advance both basic biology and therapeutic applications.

To create synthetic multivalent ligands with potentially high potencies, recombinantly produced ephrin-B2 extracellular domain was conjugated at a range of stoichiometries to highmolecular-weight hyaluronic acid (HA)-a well-characterized biopolymer present throughout the body and in particular within the brain-using 1-ethyl-3-(3-dimethylaminopropyl) carbodiimide hydrochloride (EDC)/sulfo-NHS chemistry, as described previously $^{21}$ (Fig. 1a,b). The valencies of the resulting $\sim 100 \mathrm{~nm}$ polymeric conjugates ${ }^{22}$-estimated using a bicinchoninic acid (BCA) assay and further quantified with size exclusion chromatography coupled with multi-angle light scattering (SEC-MALS; Fig. 1c)ranged from 2 to 25 ephrin molecules per $\mathrm{HA}$ chain in this synthesis.

Based on the recently discovered role of ephrin-B2 signalling in regulating neuronal lineage commitment of adult $\mathrm{NSCs}^{19}$, we investigated the activity of the multivalent conjugates in NSC culture. At a given concentration of ephrin-B2 ectodomain molecules, increasing the valency of HA:ephrin-B2 conjugates progressively elevated neuronal differentiation (Fig. 1d,e). Strikingly, compared to the antibody-clustered ligand, the 1:22, 1:12 and 1:8 valency conjugates induced similar levels of neuronal differentiation at 37-, 26- and 9-fold lower protein concentrations, respectively. Thus, in contrast to the current standard antibody-clustered form, for which the low potency necessitates high concentrations, the multivalent ligands are potent agonists, thereby potentially reducing costs. Next, the addition of monomeric ephrin-B2 in tenfold molar excess to Fc-ephrin-B2 wells blocked differentiation, further establishing that ephrin clustering is required for activity. Finally, the results were further validated by quantifying mRNA levels of the neuronal marker Tubb3 (Fig. 1f).

We next compared the ability of natural and synthetic ligands to cluster Eph receptors. Because ephrin-B2 presented from astrocytes regulates the neuronal differentiation of adult $\mathrm{NSCs}^{19}$, we analysed ephrin-Eph localization on NSCs in contact with hippocampal astrocytes. Punctate staining of both ephrin-B2 and its receptor EphB4 was observed at cell-cell junctions (Fig. 2a), and co-localization of the ligand and receptor was also observed at cell-cell contacts in the subgranular zone (SGZ) of the adult hippocampus (Fig. 2b), where NSCs reside ${ }^{19}$.

We analysed whether the multivalent conjugates could emulate this natural process of receptor-ligand assembly. Fluorescently labelled ephrin-B2 conjugates were synthesized and incubated with NSCs, at $4{ }^{\circ} \mathrm{C}$ to block endocytosis. EphB4 localization was diffuse across the cell membrane in the absence of ephrin-B2 or with low-ratio conjugates, whereas EphB4 puncta were observed in the presence of highly multivalent conjugates or antibody-clustered ligand (Fig. 2c). Additionally, although low ephrin-B2 valency conjugates yielded fewer and smaller EphB4 clusters than

\footnotetext{
'Department of Chemical and Biomolecular Engineering, 201 Gilman Hall, University of California, Berkeley, California 94720-1462, USA, ${ }^{2}$ Department of Bioengineering, 306 Stanley Hall MC \#1762, University of California, Berkeley, California 94720-1762, USA, ${ }^{3}$ Department of Material Science and Engineering, 210 Hearst Mining Building, University of California, Berkeley, California 94720-1760, USA, ${ }^{4}$ Department of Chemical and Biological Engineering, Rensselaer Polytechnic Institute, 110 8th Street, Troy, New York 12180, USA. *e-mail: schaffer@berkeley.edu
} 
a

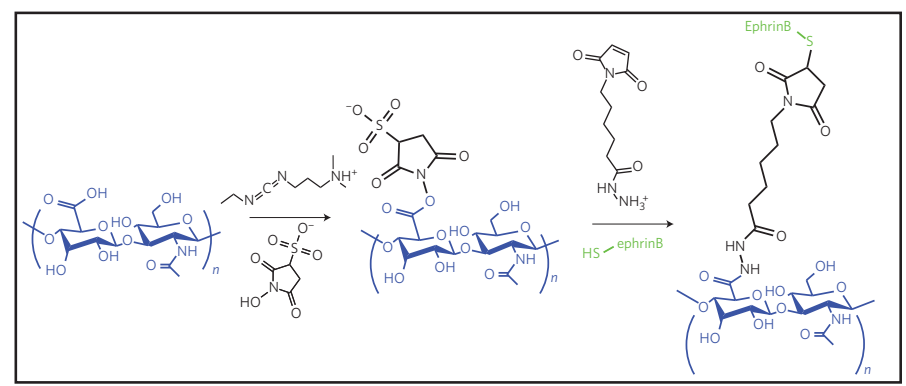

\begin{tabular}{|c|cc|}
\multicolumn{1}{c}{$\begin{array}{c}\text { Initial HA: Ephrin-B2 } \\
\text { molar ratio }\end{array}$} & \multicolumn{2}{c}{ Final ephrin-B2 molar ratio } \\
\hline $1: 0$ & BCA & SEC-MALS \\
$1: 1$ & 0.77 & 0 \\
$1: 5$ & 1.75 & 2.71 \\
$1: 10$ & 8.20 & 4.55 \\
$1: 20$ & 12.04 & 7.94 \\
$1: 40$ & 22.78 & 11.82 \\
& & \\
\hline
\end{tabular}
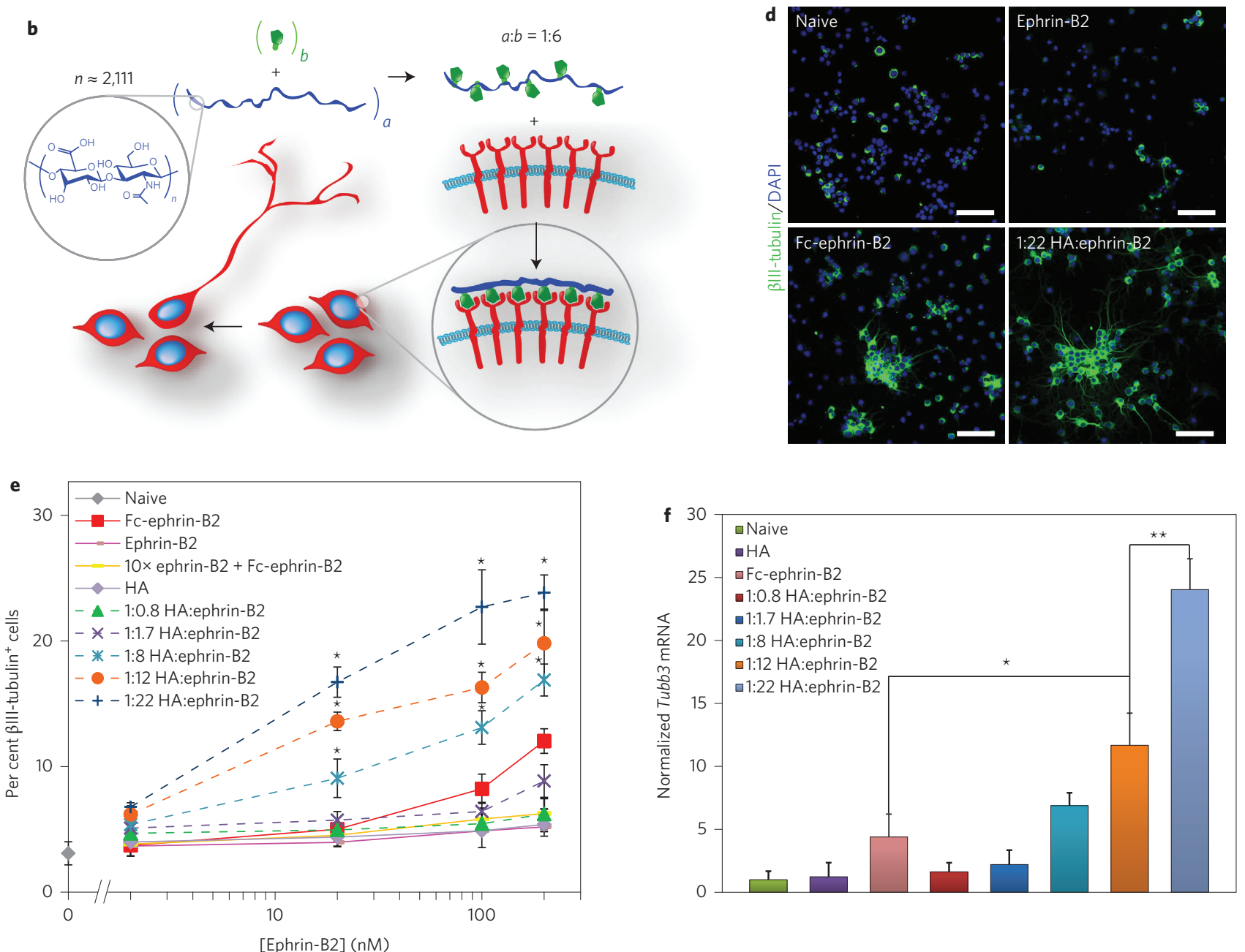

Figure 1 | Multivalent ephrin-B2 enhances neuronal differentiation of NSCs in vitro. a, From left to right: Chemical schematic of HA (blue) activation with sulfo-NHS (bottom) and EDC (top), and subsequent functionalization with EMCH (top) and recombinant protein (green) conjugation. $\mathbf{b}$, Schematic of protein (green) conjugation to $800 \mathrm{kDa}$ linear HA (blue) and subsequent clustering of receptors (red) upon introduction to cells, inducing neuronal differentiation. The molecular structure represents an HA monomer subunit, of which there are $\sim 2,111(n)$ in $800 \mathrm{kDa} H \mathrm{~A}$. The ratio $a: b$ represents the valency of HA molecules to covalently bound protein subunits. c, Comparative BCA versus SEC-MALS analysis for a range of ephrin-B2 bioconjugate valencies. d, Representative images of cultured NSCs differentiated for 6 days in media alone (naive) or in the presence of unclustered ephrin-B2, antibody clustered Fc-ephrin-B2 or multivalent 1:22 HA:ephrin-B2, then immunostained for the neuronal marker $\beta$ III-tubulin (green) and total nuclei (blue). Scale bars, $100 \mu \mathrm{m} . \mathbf{e}$, Quantification of the total fraction of neurons after 6 days of NSC differentiation in the presence of ephrin-B2 conjugates (dashed lines) or controls (solid lines), as assessed by immunostaining. ${ }^{\star} P<0.05$ compared with Fc-ephrin-B2 at corresponding ephrin-B2 concentrations. f, qPCR for the neuronal transcript Tubb3 after 6 days of differentiation. ${ }^{\star} P=0.0159,{ }^{\star}{ }^{\star} P=0.0037$. All error bars represent standard deviation from the mean.

antibody-clustered ligand, high-valency conjugates showed more (Fig. 2d), larger (Fig. 2e) and more intense (Fig. 2f) EphB4 clusters in close proximity (within the $\sim 250 \mathrm{~nm}$ resolution limit of light microscopy) to fluorescently tagged ephrin-B2. Ligand multivalency therefore modulates both the number and size of receptor clusters. In addition, we generated conjugates from ephrin-B2 protein recombinantly produced in mammalian cells and observed similar cell surface binding, indicating different protein expression systems result in similar downstream conjugate binding (Supplementary Fig. S1a).

Next, to explore the effect of ligand spacing on NSC differentiation and cell receptor clustering, monodisperse HA molecules of varying molecular weights were conjugated with recombinant ephrin-B2 extracellular domains tagged with fluorescent Alexa 

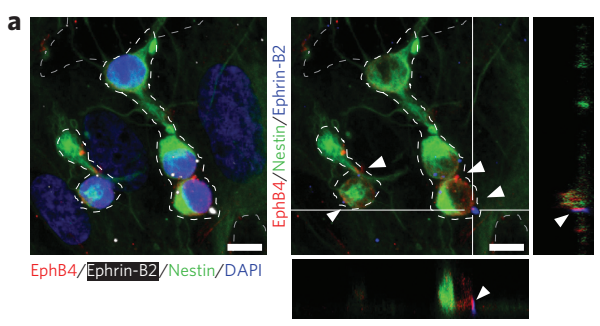
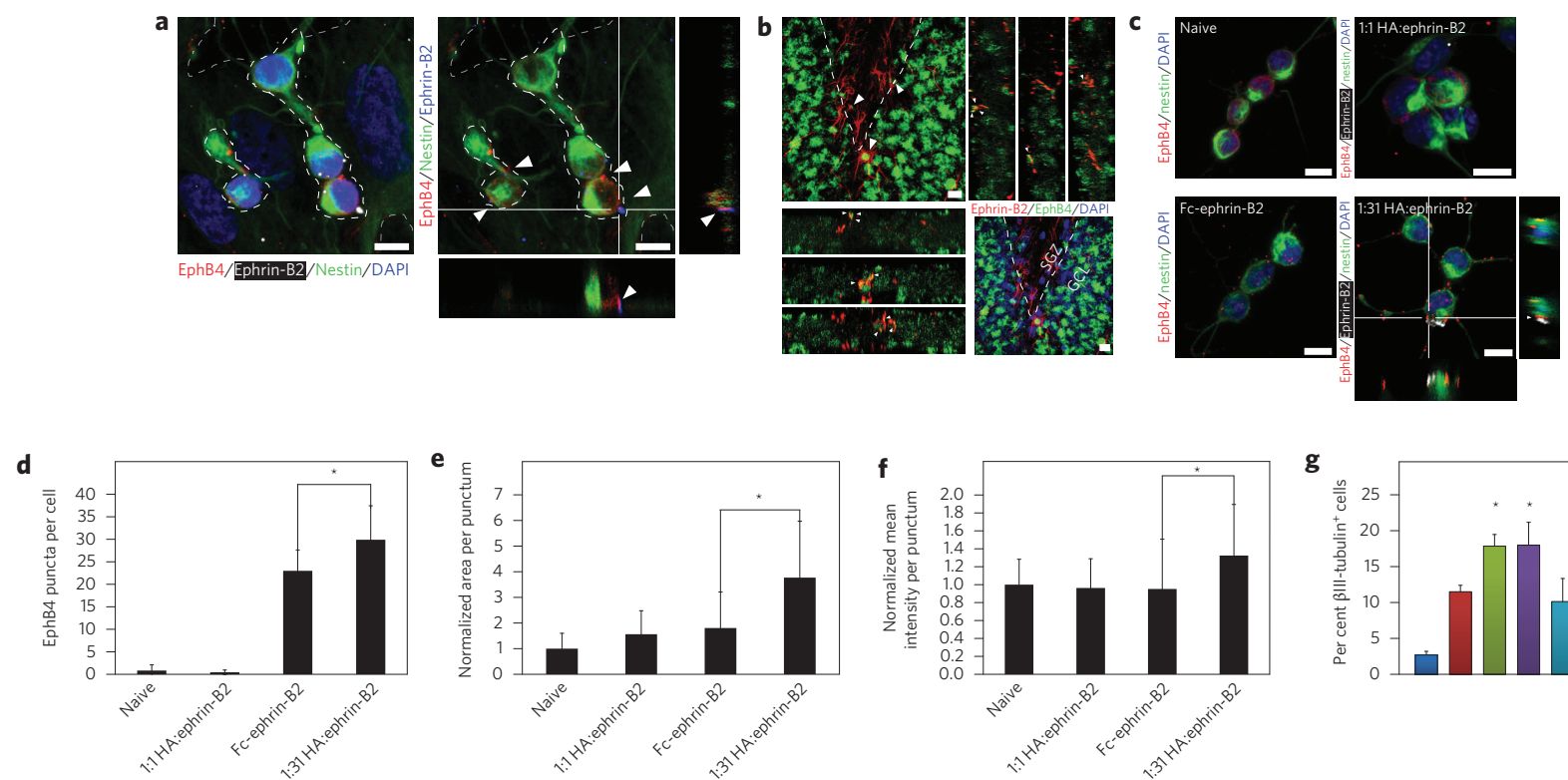

g

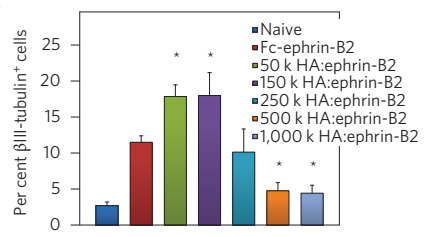

h
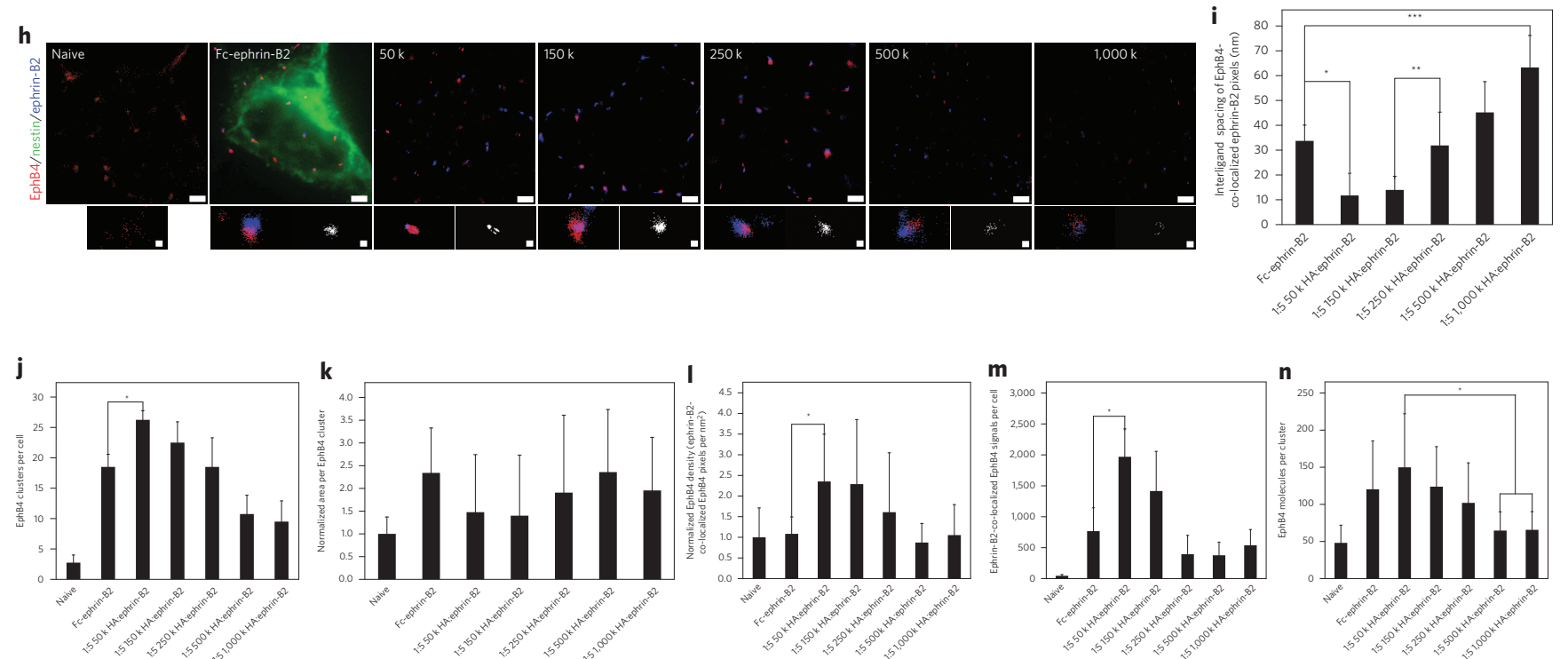

Figure 2 | Multivalent ephrin-B2 enhances receptor clustering. a, Representative image of EphB4 (red) and ephrin-B2 (white) clustering (white arrow heads) on the surface of NSCs (stained with the NSC marker nestin, pseudo-coloured green and outlined with white dashed lines) and hippocampal astrocytes (edges outlined with grey dashed lines), respectively, in co-culture. All cell nuclei are shown in blue. Orthogonal views centred at the crosshairs of the righthand $X Y$ image shown below and to the right. Scale bars, $10 \mu \mathrm{m}$. b. EphB4 (green) receptor clustering (white arrow heads) on the surface of multiple cells in the hippocampal subgranular zone (SGZ) in contact with ephrin-B2 ${ }^{+}$astrocytes (red). All cell nuclei are shown in blue. Orthogonal views centred at each of the 3 white arrow heads in the top-left XY image shown below and to the right. Scale bars, $10 \mu \mathrm{m}$. c, Representative images of NSCs incubated with fluorescently labelled ephrin-B2 bioconjugates (white) for $4 \mathrm{~h}$ at $4{ }^{\circ} \mathrm{C}$ and immunostained for their cognate receptor EphB4 (red), the NSC marker nestin (green) and total nuclei (blue). White arrow heads indicate ephrin-B2/EphB4 co-localization. Orthogonal views centred at the crosshairs of the bottom-right $X Y$ image shown below and to the right. Scale bars, $10 \mu \mathrm{m}$. d, Quantification of total number of EphB4 puncta per cell after incubation with ephrin-B2, either antibody-clustered or a new preparation of conjugate. ${ }^{\star} P=0.024$ by ANOVA, $n \geq 10$. e, Normalized area per EphB4 punctum after incubation with ephrin-B2. ${ }^{\star} P=5.33 \times 10^{-9}$ by ANOVA, $n \geq 62$. f, Normalized mean EphB4 signal intensity per punctum after incubation with ephrin-B2. ${ }^{\star} P=0.001$ by ANOVA, $n \geq 62$. , Immunostaining quantification of the total fraction of neurons after 6 days of NSC differentiation in the presence of Alexa Fluor 647 tagged ephrin-B2 conjugates of varying HA backbone molecular weight and antibody-clustered Fc-ephrin-B2 control, all at $200 \mathrm{nM}$ protein concentration. ${ }^{\star} P<0.02$ compared to Fc-ephrin-B2. h, Representative reconstructed super-resolution images of EphB4-Dendra2 (red) NSCs incubated with fluorescently labelled ephrin-B2 bioconjugates (blue) for $4 \mathrm{~h}$ at $4{ }^{\circ} \mathrm{C}$ and then immunostained for the NSC marker nestin (green). Magnified images of representative EphB4/ephrin-B2 clusters are shown below whole-cell images, with co-localized pixels indicated in white. Large scale bars, 1,000 nm; small scale bars, $100 \mathrm{~nm}$. i, Quantification of average inter-ligand spacing between individual ephrin-B2 signals that co-localized with EphB4, with a constant value of $16 \mathrm{~nm}$ per pixel. ${ }^{\star} P=1.80 \times 10^{-6},{ }^{\star \star} P=0.0006,{ }^{\star \star \star} P=1.22 \times 10^{-6}$. $\mathbf{j}$, Quantification of total number of EphB4 clusters per cell after incubation with fluorescent ephrin-B2. ${ }^{\star} P=0.0009$. $\mathbf{k}$, Normalized area per EphB4 cluster after incubation with fluorescent ephrin-B2. I, Normalized number of co-localized EphB4ephrin-B2 signals per $\mathrm{nm}^{2}$ after incubation with ephrin-B2. ${ }^{\star} P=0.0015$. $\mathbf{m}$, Total number of co-localized EphB4-ephrin-B2 signals per cell after incubation with fluorescent ephrin-B2. ${ }^{\star} P=0.0064$. $\mathbf{n}$, Total number of EphB4 signals per cluster after incubation with fluorescent ephrin-B2. ${ }^{\star} P<0.05$. All error bars represent standard deviation from the mean. 
a

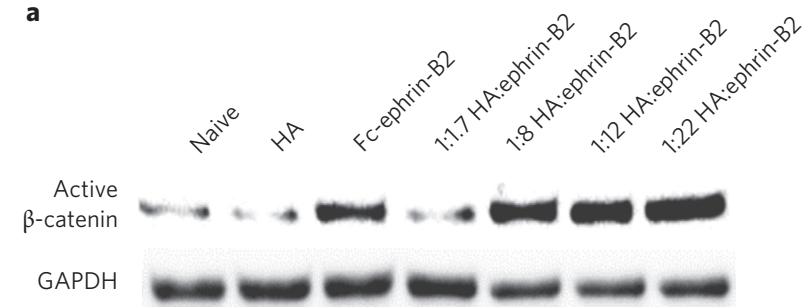

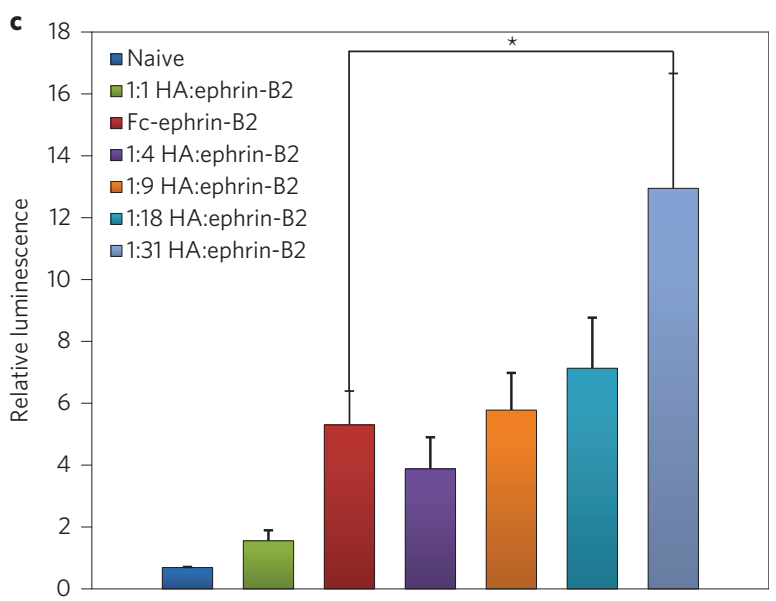
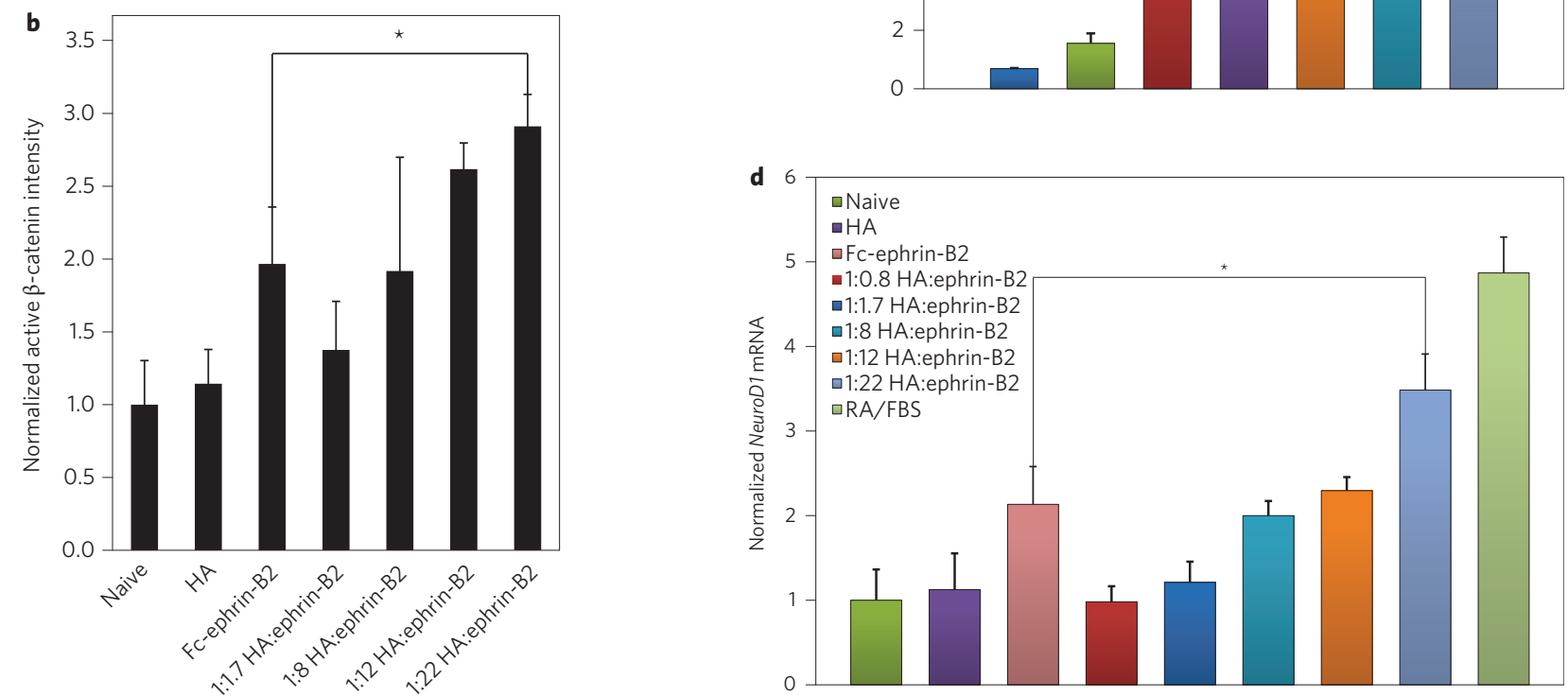

Figure 3 | Multivalent ephrin-B2 enhances downstream signalling. a, Representative western blot for active $\beta$-catenin and GAPDH, as a loading control, in NSC lysate after $24 \mathrm{~h}$ incubation with ephrin-B2 bioconjugates. $\mathbf{b}$, Average levels of $\beta$-catenin activation normalized to GAPDH in $n=3$ blots. ${ }^{\star} P=0.0217$. c, Quantification of $\beta$-catenin activation in NSCs after $24 \mathrm{~h}$ incubation with fluorescently labelled ephrin-B2 bioconjugates at $37{ }^{\circ} \mathrm{C}$, as assessed by upregulation of the transgenic luciferase reporter for $\beta$-catenin activity. ${ }^{\star} P=0.0267$. $\mathbf{d}$, qPCR for the intermediate transcriptional target of neurogenesis NeuroD1 after 6 days of differentiation. The retinoic acid/fetal bovine serum (RA/FBS) group is described in detail in the Methods. ${ }^{\star} P=0.0192$. All error bars represent standard deviation from the mean.

Fluor 647 molecules. Reactions were performed such that the polymers of varying molecular weights were linked to an equal number of fluorescently tagged proteins, with the lower-molecular-weight conjugate containing an apparently saturated number of ephrin ligands (1:5 HA:ephrin-B2 final molar ratio). The high molecular weight conjugates thus had greater inter-ligand spacing than lower-molecular-weight conjugates. After 6 days of culture, lowermolecular-weight conjugates induced significantly higher neuronal differentiation from NSCs, and higher-molecular-weight conjugates showed significantly less differentiation, compared to antibodyclustered Fc-ephrin-B2 (Fig. 2g). Inter-ligand spacing thus modulates conjugate activity.

Because standard fluorescence microscopy cannot accurately analyse the clustering properties of conjugates of different molecular weight, we applied recently developed super-resolution microscopy approaches to image receptor clusters on NSCs at $16 \mathrm{~nm}$ resolution. We generated an NSC line expressing an EphB4-Dendra $2^{23}$ fusion protein for photoactivatable localization microscopy $(\mathrm{PALM})^{24}$, which was combined with direct stochastic optical resolution microscopy (dSTORM) ${ }^{25}$ of Alexa Fluor 647-tagged HA:ephrinB2 conjugates (Fig. 2h). A single EphB4 and Ephrin-B2 signal was considered co-localized (and thus the molecules probably bound) when found to lie within the $16 \mathrm{~nm}$ resolution of the technique, corresponding to a single pixel in the images. We first analysed receptor-ligand clusters for NSCs co-cultured with astrocytes and verified the existence of punctate staining at astrocyte-NSC junctions (Supplementary Fig. S2a), and co-localization of ephrin-B2 with EphB4 was significantly more prevalent on NSCs when in contact with astrocytes (Supplementary Fig. S2b).

We next analysed clusters generated by multivalent conjugates. In the absence of ligand, EphB4 was patchy but diffuse. However, after incubation of conjugates with cells for $4 \mathrm{~h}$ at $4{ }^{\circ} \mathrm{C}$, the spacing between ephrin-B2 ligands that co-localized with EphB4 was significantly lower for the lower-molecular-weight HA conjugates (Fig. 2i). Furthermore, conjugates with closer ephrin-B2 inter-ligand spacing formed significantly more EphB4 clusters, which were also denser (that is, a higher number of co-localized EphB4-ephrin-B2 signals per $\mathrm{nm}^{2}$ ) than conjugates with larger ephrin-B2 inter-ligand spacing (Fig. 2j-1). Consequently, the shorter polymer conjugates resulted in more ligand-bound EphB4 molecules per cell (Fig. 2m) and per individual cluster (Fig. 2n). Because the number of co-localized EphB4-ephrin-B2 signals was larger than the valency of a single conjugate, each receptor-ligand cluster involved multiple bound conjugates, consistent with reports of lateral Eph receptor clustering ${ }^{26}$. Super-resolution imaging also confirmed that multivalent ligands produced in different expression systems yielded indistinguishable numbers, sizes and densities of ligand-receptor clusters (Supplementary Fig. S3a-d). 

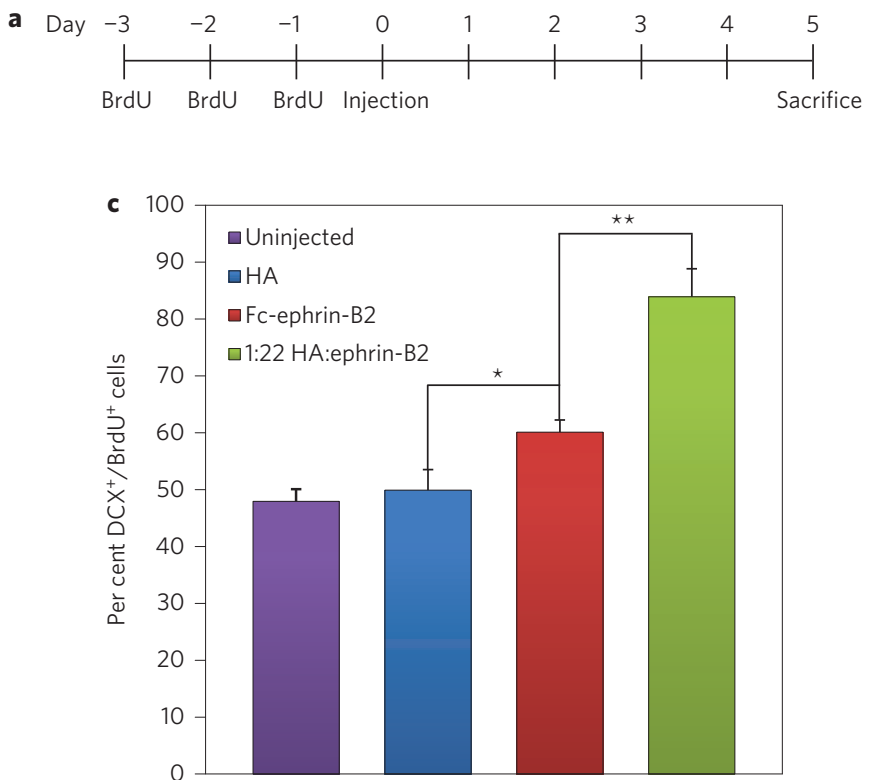
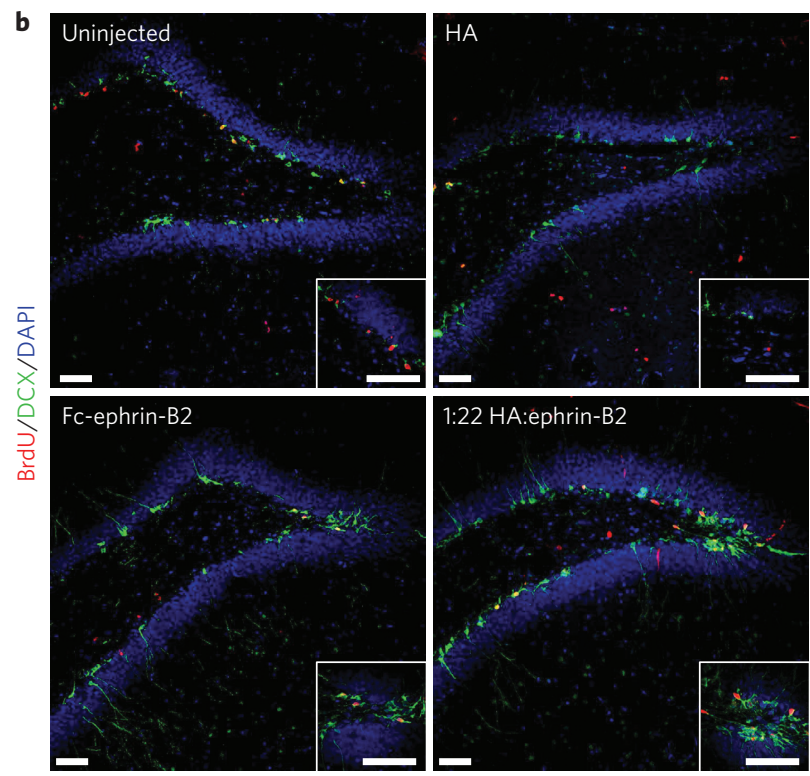

Figure 4 | Multivalent ephrin-B2 enhances in vivo neurogenesis. a, Schematic of experimental time course. b, Representative images of adult rat hippocampal sections 5 days after stereotactic injections, immunostained for BrdU (red) to label dividing cells, DCX (green) to label immature neurons, and DAPI (blue) to stain nuclei. Insets show magnified views of each image. Scale bars, $100 \mu \mathrm{m}$. c, Quantification of the overall fraction of newborn hippocampal cells that underwent neuronal differentiation using stereological estimation. ${ }^{\star} P=0.0136,{ }^{\star \star} P=0.0015$. All error bars represent standard deviation from the mean.

Modulating inter-ligand spacing can thus control the number of clusters per cell and the number of receptors per cluster, potentially due to entropic effects associated with the conjugate's polymer backbone ${ }^{27}$, and thereby control stem cell differentiation.

Ephrin-B2 induces neuronal differentiation by downstream activation of the transcriptional co-activator $\beta$-catenin ${ }^{19}$. Western blotting indicated that higher-valency conjugate activated $\beta$-catenin significantly more than antibody-clustered Fc-ephrin or HA alone (Fig. 3a,b). Furthermore, a $\beta$-catenin responsive promoter-reporter construct showed a higher quantitative level of activation with increasing valency, and at a 1:31 valency was substantially greater than antibody-clustered Fc-ephrin-B2 (Fig. 3c). Finally, $\beta$-catenin drives neuronal differentiation via transcriptional activation of the proneural transcription factor NeuroD $1^{19}$ and multivalent ephrinB2 conjugates with increasing valency again progressively induced higher levels of expression of this important target (Fig. 3d).

Multivalent conjugates may have strong utility not only in vitro but also in vivo. To investigate the latter, multivalent ephrin-B2 was administered into the hippocampal region of the adult rodent brain to analyse its ability to modulate endogenous stem cell function. Bromodeoxyuridine (BrdU) was administered to label dividing cells, followed by stereotactic injection of the ephrin-B2 conjugate or controls (Fig. 4a). After 5 days, the fractions of newly born cells $\left(\mathrm{BrdU}^{+}\right)$that had differentiated into neurons $\left(\mathrm{DCX}^{+}\right)$was quantified in tissue sections ${ }^{19}$. Antibodyclustered ligand showed a modest $20 \%$ increase over the uninjected or vehicle-injected HA control brains at the ephrin levels administered; however, the same number of ephrin-B2 domains incorporated into the highly multivalent conjugate yielded a substantial $60 \%$ increase in neurogenesis in the brain (Fig. 4b,c). These data demonstrate that nanoscale organization in the presentation of this ligand greatly enhances its ability to elicit cellular responses both in vitro and in vivo.

We next assessed the generality of this approach with both hESCs and hiPSCs. Vazin and colleagues have shown that unclustered ephrin-B1-in combination with soluble stromal cell-derived factor-1 (SDF-1), pleiotrophin (PTN) and IGF-2, a blend known as SPIE-enhanced hESC differentiation into midbrain dopaminergic neurons ${ }^{20}$, which are being considered as cell replacement therapies for Parkinson's disease ${ }^{28}$. By analogy with ephrin$\mathrm{B} 2$, we synthesized multivalent ephrin-B1 conjugates. Using conjugates of varying valency, together with the other components of SPIE, hESCs were first differentiated within embryoid bodies and then toward dopaminergic neurons for 14-18 days (Fig. 5a). After 15 days, transcripts for the midbrain-specific marker En1 increased with higher valency (Fig. 5b), and quantitative polymerase chain reaction (qPCR) showed that both the pan-neuronal marker $T u b b 3$ and the dopaminergic marker $\mathrm{TH}$ progressively increased with conjugate valency (Fig. 5c).

Immunostaining was then conducted to quantify cell differentiation into a dopaminergic lineage. After 15-18 days of differentiation in ephrin post-embryoid body formation, hESC-derived cultures exhibited substantially higher proportions of cells expressing pan-neuronal (Map2) and the dopaminergic neuron $(\mathrm{TH})$ markers with increasing conjugate valency, compared to antibodyclustered ephrin-B1 (Fig. 5d-f). Similar results were achieved with hiPSCs (Supplementary Fig. S4a,b). Because contaminating cells within dopaminergic grafts has been associated with adverse events in clinical trials ${ }^{29}$, approaches to improve dopaminergic neuron purity are significant. Finally, to assess the functional properties of the differentiated dopaminergic neurons, levels of the neurotransmitter dopamine were measured, either in conditioned medium or following the addition of $\mathrm{KCl}$ to induce synaptic neurotransmitter release. Dopamine levels increased with increasing valency and were greater with highly multivalent conjugates than antibodyclustered groups (Fig. 5g), consistent with the observed higher dopaminergic differentiation levels observed with the conjugates.

As an initial assessment of how multivalent ephrin affects differentiating cultures, at various times over 14 days cells received a $24 \mathrm{~h}$ pulse of BrdU to quantify DNA synthesis. Cells with multivalent ephrin-B1 remained mitotically active longer than with unclustered ephrin-B1 or no SPIE factors, indicating that enhanced proliferation of intermediate neuronal progenitors may increase the number of dopaminergic neurons in fully differentiated cultures (Fig. 5h). These results indicate that ephrin effects on dopaminergic differentiation are complex, and the multivalent conjugates will enable 

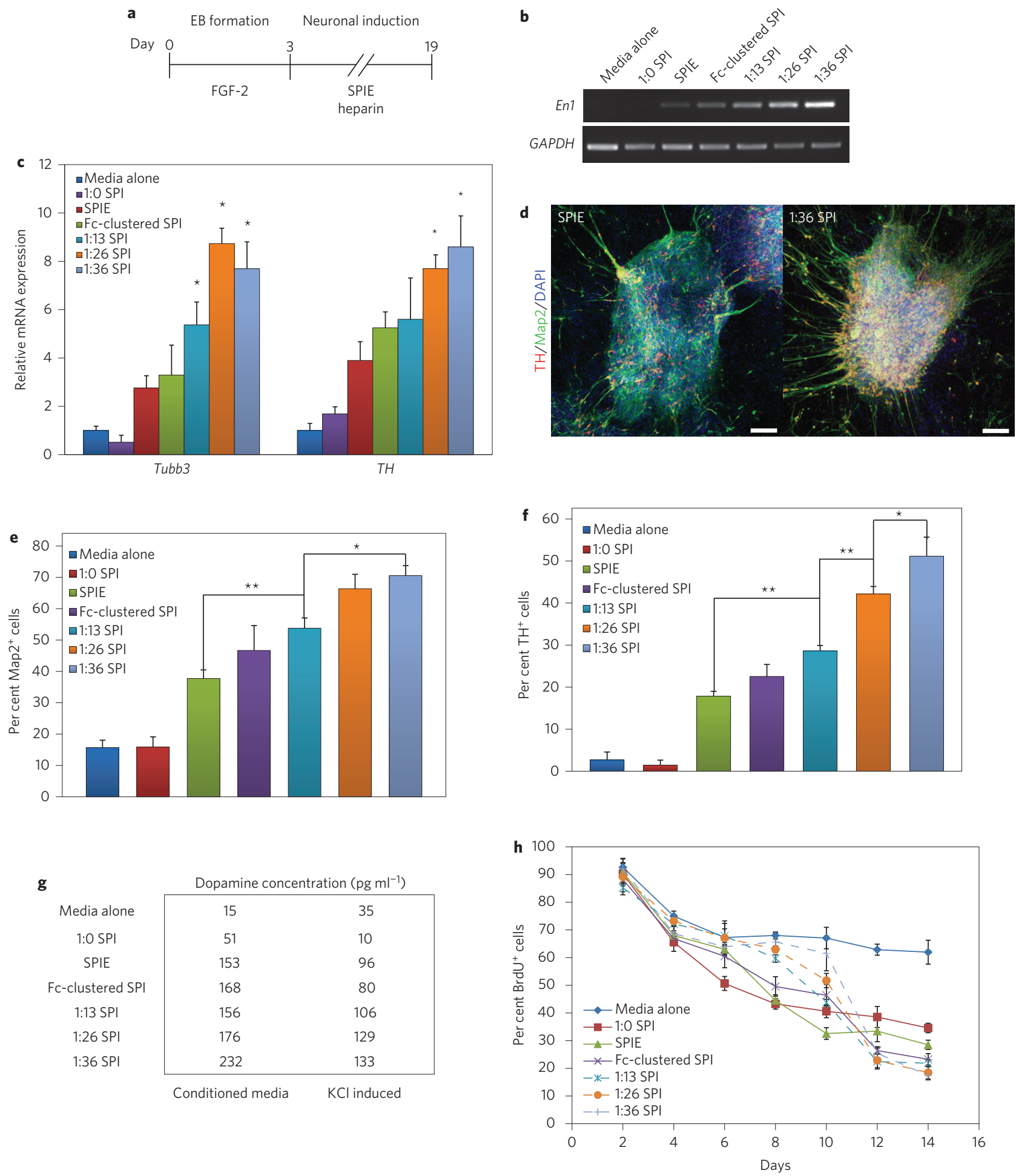

Figure 5 | Multivalent ephrin-B1 enhances neuronal and midbrain fates in differentiating hESCs. a, Schematic of experimental time course. $\mathbf{b}$, Reverse transcription polymerase chain reaction (RT-PCR) analysis of midbrain-specific marker En1 expression versus control GAPDH, from HSF6 hESCs differentiated for 15 days post-embryoid body formation with ephrin-B1 conjugates or controls. c, QPCR for the pan-neuronal marker Tubb3 and the dopaminergic marker TH after 15 days of differentiation of $\mathrm{H} 1 \mathrm{hESCs}$ with ephrin-B1 conjugates or controls. ${ }^{\star} P<0.05$ compared to SPIE for corresponding neuronal marker.

d, Representative images of cultured $\mathrm{H} 1 \mathrm{hESCs}$ differentiated for 18 days post-embryoid body formation with the SPI factors, heparin and either Fc-ephrin-B1 or multivalent 1:36 HA:ephrin-B1. Immunostaining for total neurons (green), dopaminergic neurons (red) and total nuclei (blue). Scale bars, $100 \mu \mathrm{m}$. e, Quantification of the fraction of total neurons after differentiation of $\mathrm{H} 1 \mathrm{hESCs}$ in the presence of ephrin-B1 conjugates or controls, as assessed by immunostaining. ${ }^{\star} P=0.0181,{ }^{\star}{ }^{\star} P=0.0029$. $\mathbf{f}$, Quantification of the fraction of dopaminergic neurons after differentiation of $\mathrm{H} 1 \mathrm{hESCs}$ in the presence of ephrin-B1 conjugates or controls, as assessed by immunostaining. ${ }^{\star} P=0.0328,{ }^{\star \star} P=0.0004$. $\mathbf{g}$, Quantification of total dopamine levels in cultures of $\mathrm{H} 1$ hESCs differentiated for 4 weeks in the presence of ephrin-B1 conjugates or controls, as assessed by high-performance liquid chromatography (HPLC) with electrochemical detection. $\mathbf{h}$, Quantification of the fraction of mitotic cells on various days over the 14 day course of HSF6 hESC differentiation in the presence of ephrin-B1 conjugates (dashed lines) or controls (solid lines). All error bars represent standard deviation from the mean. 
future mechanistic investigation of the role of this signalling system in dopaminergic neuron generation.

This study demonstrates that multivalency greatly enhances the bioactivity of ligands that regulate stem cell behaviour, and multivalent conjugates thus have utility for mechanistic investigation in both stem cell and developmental biology. Studying the mechanistic role of receptor complex assembly in cell-cell contact-dependent Delta-Notch ${ }^{11}$, c-kit ${ }^{12}$, Fas ligand ${ }^{13}$ and Flt ligand ${ }^{14}$ signalling-as well as matrix-binding fibroblast growth factor (FGF $)^{15}$, transforming growth factor- $\beta$ (TGF- $\beta)^{16}$, Hedgehogs $(\mathrm{Hh})^{17}$, vascular endothelial growth factor $(\mathrm{VEGF})^{18}$ signalling and other signalling systems-could greatly benefit from nanoscale synthetic ligands that potently activate receptors in a biomimetic fashion. For example, the effects of many receptor-ligand complex properties (including the number of receptor clusters per cell, cluster size, ligand scaffold, inter-receptor distance within an oligomer, and induced heteroligomerization of different receptors into the same cluster) on biological activity can now be varied systematically and studied in vitro or in vivo. Furthermore, nanoscale multivalent conjugates could potentially activate these systems more potently and thus less expensively. Finally, this platform has biotechnological and biomedical applications, including in cell culture systems, bioactive materials and drug-delivery technologies.

\section{Methods}

Recombinant protein production, purification and bioconjugation. Murine ephrin-B2 ectodomain sequence (amino acids 31-227) was amplified from the plasmid pcDNA3.1-ephrin-B2-hFc (a gift from T. Miyamoto, Keio University), and human ephrin-B1 ectodomain sequence (amino acids 28-237) was amplified from pALTER-MAX (a gift from H. Sugimura, Hamamatsu University). A C-terminal hexahistidine tag and cysteine were added during PCR, followed by insertion into the bacterial expression plasmid pBAD. Protein was expressed in bacteria and purified as described previously ${ }^{21}$. Protein purity was assessed by confirmation of a single band following SDS-polyacrylamide gel electrophoresis (SDS-PAGE). Purified ephrin-B2 or ephrin-B1 was conjugated to $800 \mathrm{kDa} H A$ (Genzyme) or to a range of monodisperse-molecular-weight HAs (Hyalose) through a two-step reaction using carbodiimide chemistry at the HA carboxylate group and a maleimide reaction at the protein C-terminal cysteine ${ }^{21}$. In the first step, $3,3^{\prime}-N-(\varepsilon$-maleimidocaproic acid) hydrazide (EMCH, Pierce, $1.2 \mathrm{mg} \mathrm{ml}^{-1}$ ), $N$-hydroxysulphosuccinimide (sulfo-NHS, Pierce, $2.8 \mathrm{mg} \mathrm{ml}^{-1}$ ) and 1-ethyl-3-(3-dimethylaminopropyl) carbodiimide hydrochloride (EDC, Pierce, $10 \mathrm{mg} \mathrm{ml}^{-1}$ ) were added to a $3 \mathrm{mg} \mathrm{ml}^{-1}$ solution of HA in 0.1 M 2-( $N$-morpholino)ethanesulphonic acid (MES) (Sigma) buffer (pH 6.5) and allowed to react at $4{ }^{\circ} \mathrm{C}$ for $4 \mathrm{~h}$. The solution was then dialysed into pH 7.0 PBS containing 10\% glycerol and 2 mM EDTA. Recombinant ephrin-B2 or ephrin-B1 was reduced using tris(2-carboxyethyl)phosphine hydrochloride (TCEP) (Pierce) in 200 -fold molar excess and reacted at $4{ }^{\circ} \mathrm{C}$ for $5 \mathrm{~min}$. Activated HA$\mathrm{EMCH}$ was then added at desired molar ratios with reduced ephrin-B2 or ephrin-B1 and allowed to react at $4{ }^{\circ} \mathrm{C}$ overnight. The ephrin-conjugated $\mathrm{HA}$ was dialysed with $100 \mathrm{kDa}$ molecular weight cut-off (MWCO) tubing (Spectrum Labs) in pH 7.0 PBS containing $2 \mathrm{mM}$ EDTA to remove unreacted ephrin. Purified ephrin and HAconjugated ephrin protein concentrations were measured using a BCA assay, and valencies were verified using SEC-MALS as described previously ${ }^{21}$.

Antibody-clustered ephrin-B formation. To create clustered ephrin-B2 and ephrin-B1 complexes (Fc-ephrin-B), recombinant mouse ephrin-B/Fc chimera (R\&D Systems) was incubated with goat anti-human IgG, Fc-fragment specific (Jackson ImmunoResearch) antibody at a 1:9 ratio (wt/wt), which led to maximal activity of the resulting clusters (data not shown). After $90 \mathrm{~min}$ at $4{ }^{\circ} \mathrm{C}$, complexes were used immediately.

Cell culture and differentiation. NSCs were cultured as described previously ${ }^{30}$. For differentiation studies, eight-well chamber slides were seeded with $2 \times 10^{4}$ cells per well in standard culture medium containing $0.1 \mathrm{ng} \mathrm{ml}^{-1}$ FGF-2. For the subsequent 6 days, ephrin-B2 was added at the desired molar concentration and daily $50 \%$ media changes were performed. EphB4 cluster properties were quantified using ImageJ.

Immunostaining. Coronal brain sections $(40 \mu \mathrm{m})$ were processed, stored and stained as described previously ${ }^{31}$. Sections were then mounted on glass slides and either stereological analysis (Stereo Investigator, MBF Biosciences) or confocal microscopy (LSM 710, Zeiss) was performed. In short, using an optical fractionator method, $\mathrm{BrdU}^{+}$and $\mathrm{DCX}^{+}$cells were counted in a systematic randomly sampled (SRS) set of unbiased virtual volumes inside the subgranular zone and granular cell layer of both the left and right sides of the hippocampus. An estimate of the total number of $\mathrm{DCX}^{+} / \mathrm{BrdU}^{+}$cells in the hippocampus was then generated.
Super-resolution microscopy. Cultures incubated with fluorescent conjugate were fixed and in some cases immunostained with Alexa 647 or Alexa 488 tagged antibodies, then incubated with $100 \mathrm{~nm}$ Tetraspeck fluorescent beads (Life Technologies) in PBS at a 1:2,000 ( $\mathrm{vol} / \mathrm{vol})$ dilution to act as reference points during imaging to later use for drift correction in image reconstruction. The sample coverslips were then placed in an Attofluor cell chamber (Life Technologies) and incubated in cold, freshly made $\mathrm{pH} 8.0$ buffer containing $100 \mathrm{mM}$ cysteamine (Sigma), $50 \mathrm{mM}$ Tris, $10 \mathrm{mM} \mathrm{NaCl}, 10 \%$ (wt/vol) glucose, $560 \mu \mathrm{g} \mathrm{ml}^{-1}$ glucose oxidase (Sigma) and $34 \mu \mathrm{g} \mathrm{ml}^{-1}$ catalase (Sigma). Using a microscope (TE2000 Nikon) with adaptive optics for three-dimensional localization ${ }^{32}$, a piezoelectric stage and highly inclined thin illumination capability ${ }^{33}$, photoactivatable localization microscopy (PALM) ${ }^{24}$ was performed to detect EphB4-Dendra $2^{23}$ simultaneously with direct stochastic optical reconstruction microscopy (dSTORM) ${ }^{25}$ to detect Alexa Fluor 647-tagged ephrin-B2 conjugates, as described previously ${ }^{24,25}$. Subdiffraction limit images were then reconstructed using QuickPALM and quantified using ImageJ. To detect individual ephrin-B2 signals that co-localized with EphB4 signals, individual channel intensities were maximized, merged, then made into a binary image to display only co-localized pixels, indicating a proximity of signals within $16 \mathrm{~nm}$. EphB4 or ephrin-B2 ${ }^{+}$clusters were defined as any grouping of 10 or more positive pixels within a total diameter of 10 to 100 pixel widths.

Statistical analysis. The statistical significance of the results was determined using analysis of variance (ANOVA) and a multiple means comparison function (TukeyKramer method) in MATLAB with an alpha level of 0.05. All error bars are reported in standard deviation from the mean, with $n=3$ unless otherwise noted.

See Supplementary Information for Supplementary Methods.

Received 15 November 2012; accepted 11 September 2013; published online 20 October 2013

\section{References}

1. Jiang, W., Kim, B. Y., Rutka, J. T. \& Chan, W. C. Nanoparticle-mediated cellular response is size-dependent. Nature Nanotech. 3, 145-150 (2008).

2. Mannix, R. J. et al. Nanomagnetic actuation of receptor-mediated signal transduction. Nature Nanotech. 3, 36-40 (2008).

3. McMurray, R. J. et al. Nanoscale surfaces for the long-term maintenance of mesenchymal stem cell phenotype and multipotency. Nature Mater. 10, 637-644 (2011).

4. Ng, Q. K. et al. Engineering clustered ligand binding into nonviral vectors: $\alpha_{\mathrm{v}} \beta_{3}$ targeting as an example. Mol. Ther. 17, 828-836 (2009).

5. Maheshwari, G., Brown, G., Lauffenburger, D. A., Wells, A. \& Griffith, L. G. Cell adhesion and motility depend on nanoscale RGD clustering. J. Cell Sci. 113, 1677-1686 (2000).

6. Lee, L. A. et al. Multivalent ligand displayed on plant virus induces rapid onset of bone differentiation. Mol. Pharmacol. 9, 2121-2125 (2012).

7. Petrie, T. A. et al. Multivalent integrin-specific ligands enhance tissue healing and biomaterial integration. Sci. Transl. Med. 2, 45 ra60 (2010).

8. Mammen, M., Choi S-K. \& Whitesides, G. M. Polyvalent interactions in biological systems: implications for design and use of multivalent ligands and inhibitors. Angew. Chem. Int. Ed. 37, 2754-2794 (1998).

9. Kiessling, L. L., Gestwicki, J. E. \& Strong, L. E. Synthetic multivalent ligands in the exploration of cell-surface interactions. Curr. Opin. Chem. Biol. 4, 696-703 (2000)

10. Vance, D., Shah, M., Joshi, A. \& Kane, R. S. Polyvalency: a promising strategy for drug design. Biotechnol. Bioeng. 101, 429-434 (2008).

11. Artavanis-Tsakonas, S., Rand, M. D. \& Lake, R. J. Notch signaling: cell fate control and signal integration in development. Science 284, 770-776 (1999).

12. Broudy, V. C., Lin, N. L., Buhring, H. J., Komatsu, N. \& Kavanagh, T. J. Analysis of c-kit receptor dimerization by fluorescence resonance energy transfer. Blood 91, 898-906 (1998).

13. Holler, N. et al. Two adjacent trimeric Fas ligands are required for Fas signaling and formation of a death-inducing signaling complex. Mol. Cell. Biol. 23, 1428-1440 (2003)

14. Barleon, B. et al. Mapping of the sites for ligand binding and receptor dimerization at the extracellular domain of the vascular endothelial growth factor receptor FLT-1. J. Biol. Chem. 272, 10382-10388 (1997).

15. Ye, S. et al. Structural basis for interaction of FGF-1, FGF-2, and FGF-7 with different heparan sulfate motifs. Biochemistry 40, 14429-14439 (2001).

16. Haudenschild, D. R. et al. Enhanced activity of TGF- $\beta 1$ bound to cartilage oligomeric matrix protein. J. Biol. Chem. 286, 43250-43258 (2011).

17. Chang, S. C., Mulloy, B., Magee, A. I. \& Couchman, J. R. Two distinct sites in sonic hedgehog combine for heparan sulfate interactions and cell signaling functions. J. Biol. Chem. 286, 44391-44402 (2011).

18. Krilleke, D., Ng, Y. S. \& Shima, D. T. The heparin-binding domain confers diverse functions of VEGF-A in development and disease: a structure-function study. Biochem. Soc. Trans. 37, 1201-1206 (2009).

19. Ashton, R. S. et al. Astrocytes regulate adult hippocampal neurogenesis through ephrin-B signaling. Nature Neurosci. 15, 1399-1406 (2012). 
20. Vazin, T. et al. A novel combination of factors, termed SPIE, which promotes dopaminergic neuron differentiation from human embryonic stem cells. PLoS ONE 4, e6606 (2009).

21. Wall, S. T. et al. Multivalency of Sonic hedgehog conjugated to linear polymer chains modulates protein potency. Bioconj. Chem. 19, 806-812 (2008).

22. Pollock, J. F., Ashton, R. S., Rode, N. A., Schaffer, D. V. \& Healy, K. E. Molecular characterization of multivalent bioconjugates by size-exclusion chromatography with multiangle laser light scattering. Bioconj. Chem. 23, 1794-1801 (2012).

23. Gurskaya, N. G. et al. Engineering of a monomeric green-to-red photoactivatable fluorescent protein induced by blue light. Nature Biotechnol. 24, 461-465 (2006).

24. Betzig, E. et al. Imaging intracellular fluorescent proteins at nanometer resolution. Science 313, 1642-1645 (2006).

25. Van de Linde, S. et al. Direct stochastic optical reconstruction microscopy with standard fluorescent probes. Nature Protoc. 6, 991-1009 (2011).

26. Pasquale, E. B. Eph receptor signalling casts a wide net on cell behaviour. Nature Rev. Mol. Cell Biol. 6, 462-475 (2005).

27. Kane, R. S. Thermodynamics of multivalent interactions: influence of the linker. Langmuir 26, 8636-8640 (2010).

28. Lindvall, O. Dopaminergic neurons for Parkinson's therapy. Nature Biotechnol. 30, 56-58 (2012).

29. Politis, M. et al. Serotonergic neurons mediate dyskinesia side effects in Parkinson's patients with neural transplants. Sci. Transl. Med. 2, 38ra46 (2010)

30. Lai, K., Kaspar, B. K., Gage, F. H. \& Schaffer, D. V. Sonic hedgehog regulates adult neural progenitor proliferation in vitro and in vivo. Nature Neurosci. 6, 21-27 (2003)

31. Kempermann, G., Kuhn, H. G. \& Gage, F. H. More hippocampal neurons in adult mice living in an enriched environment. Nature 386, 493-495 (1997).

32. Zawadzki, R. J. et al. Adaptive-optics optical coherence tomography for highresolution and high-speed 3D retinal in vivo imaging. Opt. Express 13, 8532-8546 (2005).
33. Tokunaga, M., Imamoto, N. \& Sakata-Sogawa, K. Highly inclined thin illumination enables clear single-molecule imaging in cells. Nature Methods 5, 159-161 (2008).

\section{Acknowledgements}

The authors thank A. Ciesielska (Bankiewicz Lab, UCSF) for help with HPLC analysis of dopamine and J. Martin (RPI) for suggestions regarding the synthesis of multivalent ligands based on monovalent hyaluronic acid scaffolds. This work was supported by the National Institutes of Health (NIH R21 EB007295) and the California Institute for Regenerative Medicine (CIRM) (grant RT2-02022). A.C. and T.V. were partially supported by training grant fellowships from CIRM (T1-00007). D.P.S. was partially supported by a National Science Foundation Graduate Research Fellowship and a training grant fellowship from CIRM (TG2-01164).

\section{Author contributions}

A.C. performed all the experiments and analysed all data. A.C. and D.V.S. designed the ephrin-B2 experiments and wrote the manuscript. T.V., A.C. and D.V.S. designed the ephrin-B1 experiments. D.P.S. cloned the EphB4-Dendra2 retroviral vector. N.R. conducted the SEC-MALS experiment. K.E.H. and R.S.K. provided critical feedback on the manuscript.

\section{Additional information}

Supplementary information is available in the online version of the paper. Reprints and permissions information is available online at www.nature.com/reprints. Correspondence and requests for materials should be addressed to D.V.S.

\section{Competing financial interests}

K.E.H. is an inventor of intellectual property related to HA bioconjugates. T.V. is an inventor of intellectual property related to dopaminergic differentiation of hESCs using SPIE. 